\title{
Effect of Confinement on the Crystallization of a Dusty Plasma in Narrow Channels
}

\author{
B. A. Klumov and G. E. Morfill \\ Max Planck Institut für Extraterrestrische Physik, D-85740 Garching, Germany \\ Received March 6, 2008
}

\begin{abstract}
Three-dimensional quasi-equilibrium configurations of a complex (dusty) plasma in narrow channels are investigated using the molecular dynamics simulations for various confining potentials (confinements). The dynamics of the microparticles is described within the framework of a Langevin thermostat with allowance for the pair interaction between charged particles, which is described by a screened Coulomb potential (Yukawa potential). Two confinements - the parabolic potential and hard elastic wall-are considered. It is shown that the confinement strongly affects the crystallization and the local order of the microparticles in the system under consideration; in particular, the appearance of a new quasicrystalline phase induced by the hard wall confinement is revealed.

PACS numbers: 52.27.Lw, 61.20.Ja, 64.60.Cr

DOI: $10.1134 / \mathrm{S} 0021364008080043$
\end{abstract}

Interest in processes in a plasma containing microparticles has increased sharply in recent years. Such a plasma is called the complex plasma or dusty plasma [1-5]. First, the complex plasma is of interest due to its great abundance in nature. Interstellar clouds, protostellar clusters, planetary rings [6], comet atmospheres [7], and the ionospheres [8] and magnetospheres of planets (e.g., noctilucent clouds in the Earth's ionosphere [9]) are complex plasmas to a certain extent. Second, the possibility of observing the behavior of a single microparticle provides the kinetic, most detailed, description of the properties of the ensemble of dust particles. Owing to these circumstances, the dusty plasma is an attractive tool for studying various fundamental physical problems such as phase transitions [5], hydrodynamic instabilities [10], properties of crystallization waves $[11,12]$, etc. One of these problems is the behavior of charged micro- and nanoparticles in narrow channels when the interparticle distance is comparable with the channel width; in this case, the effect of the walls on the phase state of microparticles can be very important (see, e.g., [13]). The features of the flow of charged fluids in capillaries [14], the investigation of confinement-induced phase transitions (see, e.g., [15, 16]), the physics of nanofluids [17], the permeability of ion channels in biophysics [18], and the effect of the confinement on the phase state of granular media (see, e.g., [19]) are among the problems for which investigations of the complex plasma can be very informative.

The complex/dusty plasma is usually created in laboratories by adding microparticles to a weakly ionized rf plasma of low-pressure inert gases. The recombination of electrons and ions on the surface of dust particles lead to their fast charging, and the charge value depends on the size of a particle and plasma parameters. In particular, the $1-\mu \mathrm{m}$ particle acquires a charge of $Z_{\mathrm{d}} / e \sim 10^{3}$, where $e$ is the elementary charge, in a typical RF discharge in argon. Such a large charge of the microparticle often leads to the strong nonideality of the dust component, which can be in a wide range of phase states, i.e., can behave as a gas, liquid, or crystal. The crystalline state of the dust component of the complex plasma (so-called plasma crystal) was experimentally discovered in $1994[20,21]$ and was theoretically predicted as early as in 1986 [22].

Owing to the fast diffusion of electrons on the walls of the discharge chamber, the central region of the discharge is positively charged and is a potential well for negatively charged microparticles. The profile of the confining potential (confinement) $\Phi_{\mathrm{c}}$ near the center can be considered as parabolic, $\Phi_{\mathrm{c}}(x) \propto\left(x-x_{\mathrm{c}}\right)^{2}$, where $x_{\mathrm{c}}$ is the center of the discharge region. ${ }^{1}$ The confinement was measured, e.g., in [23], where it was shown that the confinement is close to the parabolic shape in the central discharge region. The electric field in the near-electrode region of the discharge increases much more strongly than in the bulk, and the confinement is close to the hard wall.

This work is primarily devoted to the local order of microparticles in three-dimensional narrow channels for two confinements, the parabolic and hard wall. The pair interaction between microparticles is described by the screened Coulomb potential (Yukawa potential) $\phi(r)=\left(Z_{\mathrm{d}} / r\right) \exp \left(-r / \lambda_{\mathrm{D}}\right)$, where $r$ is the distance between

\footnotetext{
${ }^{1}$ Various tools such as metallic rings, special topology of electrodes, etc. can be additionally used to confine the dust component.
} 


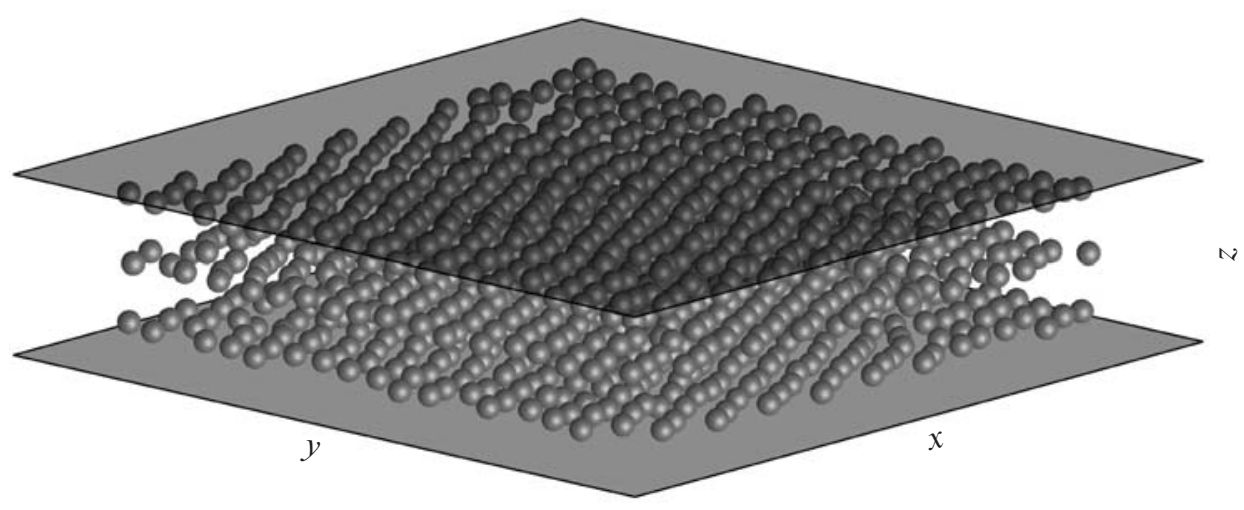

Fig. 1. Geometry of the problem under consideration. At the initial time, $N$ microparticles with the charge $Z_{\mathrm{d}}$ are randomly distributed in the space between two planes. The confinement limits the $z$ coordinate of the microparticle $\left(0 \leq z \leq L_{z}\right)$, whereas in the $(x, y)$ plane, the particles are located in the region $L_{x} \times L_{y}: 0 \leq x, y \leq L_{x, y}$.

the particles and $\lambda_{D}$ is the screening length (note that the interparticle-interaction potential measured in [23] is close to the Yukawa potential). Since we analyze the features of the crystallization of the dusty subsystem, the complex plasma is in the strongly coupled state. This means that the nonideality parameter of the dusty

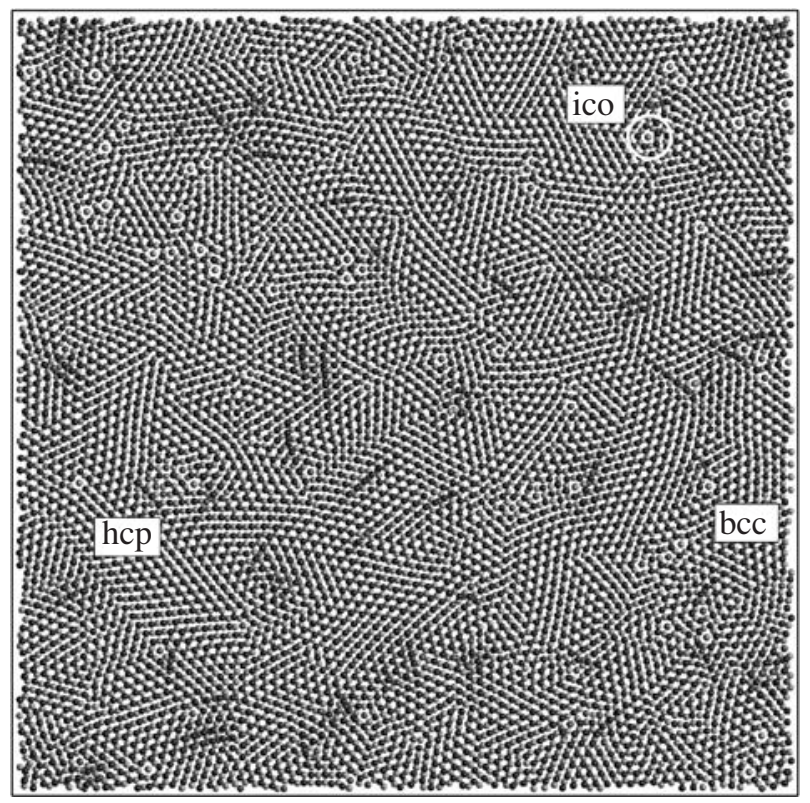

Fig. 2. Crystallized Yukawa system of microparticles in the narrow channel for the parabolic confinement. The microparticles form three layers A, B, and C. The particles of layers $\mathrm{A}, \mathrm{B}$, and $\mathrm{C}$ are marked by black, gray, and white, respectively. It is seen that layer $\mathrm{C}$ is almost completely screened by layer A. The hcp lattice is the dominant crystallattice type in this case. The bcc phase is also seen. A small number of clusters $(\sim 1 \%)$ have icosahedral (fivefold) symmetry. subsystem is large, $\Gamma=Z_{\mathrm{d}}^{2} \exp (-\kappa) / T_{\mathrm{d}} \Delta \gg 1$, where $T_{\mathrm{d}}$ is the temperature of the microparticles and $\kappa=\Delta / \lambda_{\mathrm{D}}$ is the so-called structure parameter.

The effect of the confinement on the equilibrium configurations of the dusty component of the complex plasma (pair interaction between dust particles was described by the Yukawa potential) in narrow channels in the two-dimensional geometry was considered in [24]. In particular, it was shown that the confinement strongly affects the local order of the microparticles in the system; for example, the presence of the hard wall confinement induces the transition of the microparticles to the glassy state at large $\Gamma$ values due to the increased density of microparticles near the wall, whereas a plasma crystal is observed for the parabolic confinement. Below, we consider a similar problem, but in three-dimensional geometry.

The behavior of the ensemble of the microparticles in narrow three-dimensional channels is investigated using the molecular dynamics method. For simplicity, we assume that all microparticles have the same charge $Z_{\mathrm{d}}$ and the pair interaction between dust particles is described by the Yukawa potential. The equation of motion of an individual microparticle has the form

$$
m \ddot{\mathbf{r}}_{i}=-Z_{\mathrm{d}} \nabla \Phi_{\mathrm{c}}-Z_{\mathrm{d}} \sum \nabla \phi-m \gamma \dot{\mathbf{r}}_{i}+\mathbf{L}_{i}
$$

The term on the right-hand side of Eq. (1) describes the electrostatic interaction between the particles, the drag of the dust particles due to collisions with neutral atoms and molecules of the buffer gas (neutral drag), the random Langevin force $\mathbf{L}_{i}$ (thermal noise induced by neutral particles), which is determined from the relation $\left\langle\mathbf{L}_{i}(t) \mathbf{L}_{j}(t+\tau)\right\rangle=2 \gamma m k_{\mathrm{B}} \delta_{i j} \delta(\tau)$ under the condition $\left\langle\mathbf{L}_{i}(t)\right\rangle=0$, and the interaction of the microparticles with the confinement potential $\Phi_{\mathrm{c}}$.

System (1) is solved for $N=16000$ microparticles, which are randomly distributed in a narrow channel at the initial time. Figure 1 shows the geometry of the 


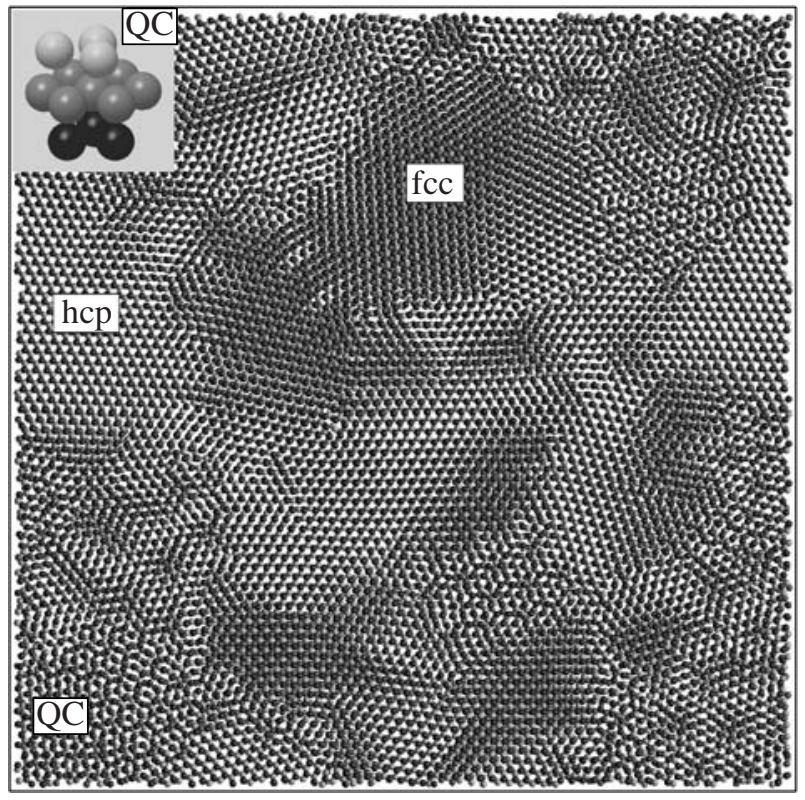

Fig. 3. Crystallization of the microparticles in the narrow channel for the hard-wall confinement. The regions with hcp and fcc crystal lattices are clearly seen. A noticeable number of clusters have a quasicrystalline phase. The inset shows the unit cell of the quasicrystalline phase, which is a distorted hcp/fcc unit cell.

problem under consideration. The confinement limits the position of the microparticles along the $z$ axis $(0 \leq$ $\left.z \leq L_{z}\right)$, whereas in the $(x, y)$ plane, the particles are located in the region $0 \leq x, y \leq L_{x, y}$. The periodic boundary conditions are used on the lateral edges $\left(x=\left\{0, L_{x}\right\}\right.$, $\left.y=\left\{0, L_{y}\right\}\right)$. We use two confinements, parabolic $\Phi_{\mathrm{c}}(z) \propto\left(z-L_{z} / 2\right)^{2}$ and hard wall $\Phi_{\mathrm{c}}(z) \propto \exp \left(\frac{z-L_{z}}{\Delta_{\mathrm{w}}}\right)$ for $z>L_{z}$ and $\Phi_{\mathrm{c}}(z) \propto \exp \left(-z / \Delta_{\mathrm{w}}\right)$ for $z<0$, where the spatial scale $\Delta_{\mathrm{w}}$ determines the hardness of the wall. The value $\Delta_{\mathrm{w}} \simeq \Delta / 3$ is used in our calculations.

It is known that an infinite system of particles whose pair interaction is described by the Yukawa potential in the thermodynamic limit for various nonideality parameters $\Gamma$ can have only two types of a crystal lattice: fcc for large values of the structure parameter $\kappa$ and bcc for small $\kappa$ values (see, e.g., [5]). The hcp phase can be formed in the nonequilibrium Yukawa system (see, e.g., [12]). This is due to the closeness of the energies required for the formation of the hcp and fcc phases [25]. To identify the fcc/hcp/bcc lattice types, it is sufficient to know the positions of three neighboring layers A, B, and C. In this case, the type of the appearing crystal lattice can be determined visually. For this reason, we consider the behavior of a Yukawa system consisting of three layers.

Some simulation results for the Yukawa system are presented in Figs. 2 and 3 for the parabolic and hard-

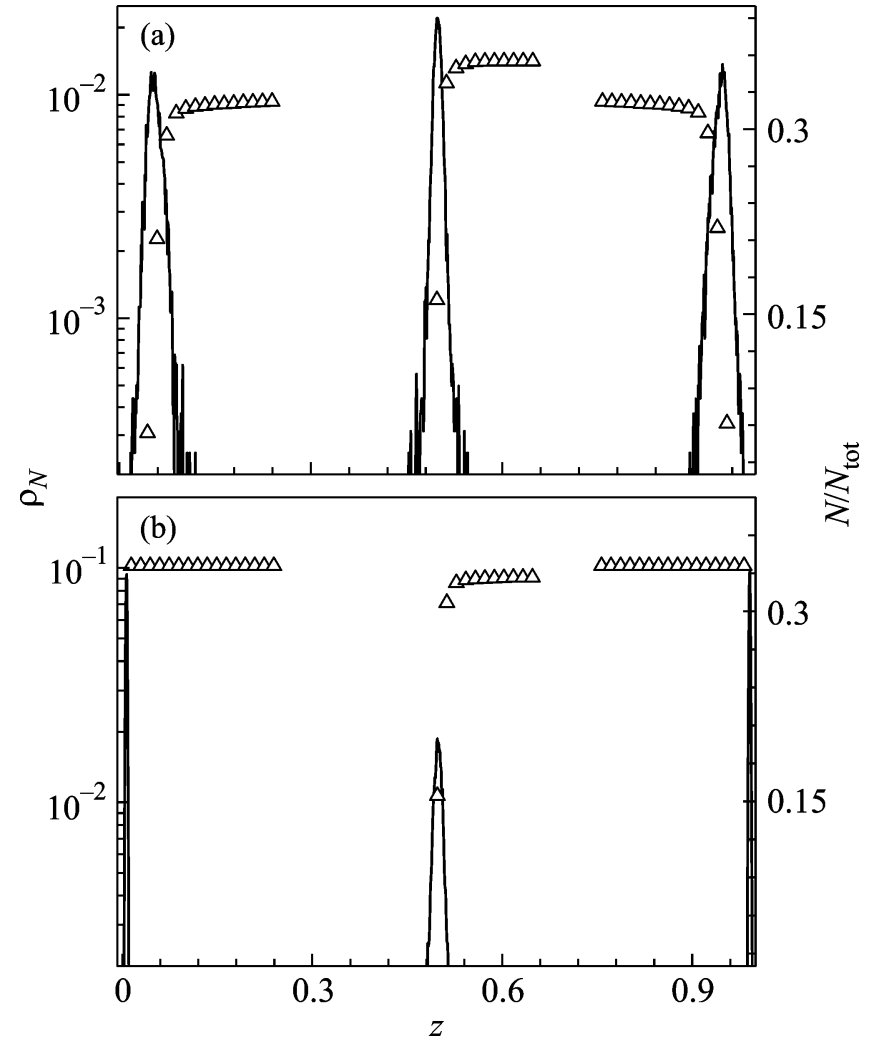

Fig. 4. Relative density of the microparticles $\rho_{N}$ and (open triangles) the total number of particles $N / N_{\text {tot }}$ in each layer versus the dimensionless coordinate $\tilde{z} \equiv z / L_{z}$ for the (a) parabolic and (b) hard elastic wall confinements.

wall confinements, respectively, where the positions of the microparticles in the $(x, y)$ plane are shown (all three layers of the particles are given). The calculations were performed with the following system parameters: the size and charge of the microparticles are $a \simeq 1 \mu \mathrm{m}$ and $Z_{\mathrm{d}} / e \simeq 3 \times 10^{3}$, respectively; $\kappa \simeq 2-3$; and the neutral gas density is $\rho_{\mathrm{g}} \sim 10^{-7} \mathrm{~g} / \mathrm{cm}^{3}$. Both figures demonstrate the quasi-stationary crystalline phase of the Yukawa system at the nonideality parameter $\Gamma \sim 10^{4}$. In both figures, the particles are color coded by $z$. It is seen that the parabolic confinement leads primarily to the formation of crystallites with the ABA layer arrangement (the third layer is screened by the first layer), which is characteristic of the hcp or bcc phase. A relatively small number of clusters $(\sim 1 \%)$ have icosahedral symmetry; a microparticle from the central layer has five neighbors near the system boundary (fivefold symmetry). Note that this lattice type is induced by the confinement. The regions with the ABA (hcp/bcc phases) and $\mathrm{ABC}$ (particles of three layers are seen) layer arrangements are clearly seen in Fig. 3 for the hard elastic wall confinement. A noticeable number of dust particles have fcc and hcp crystal lattices. The appearance 


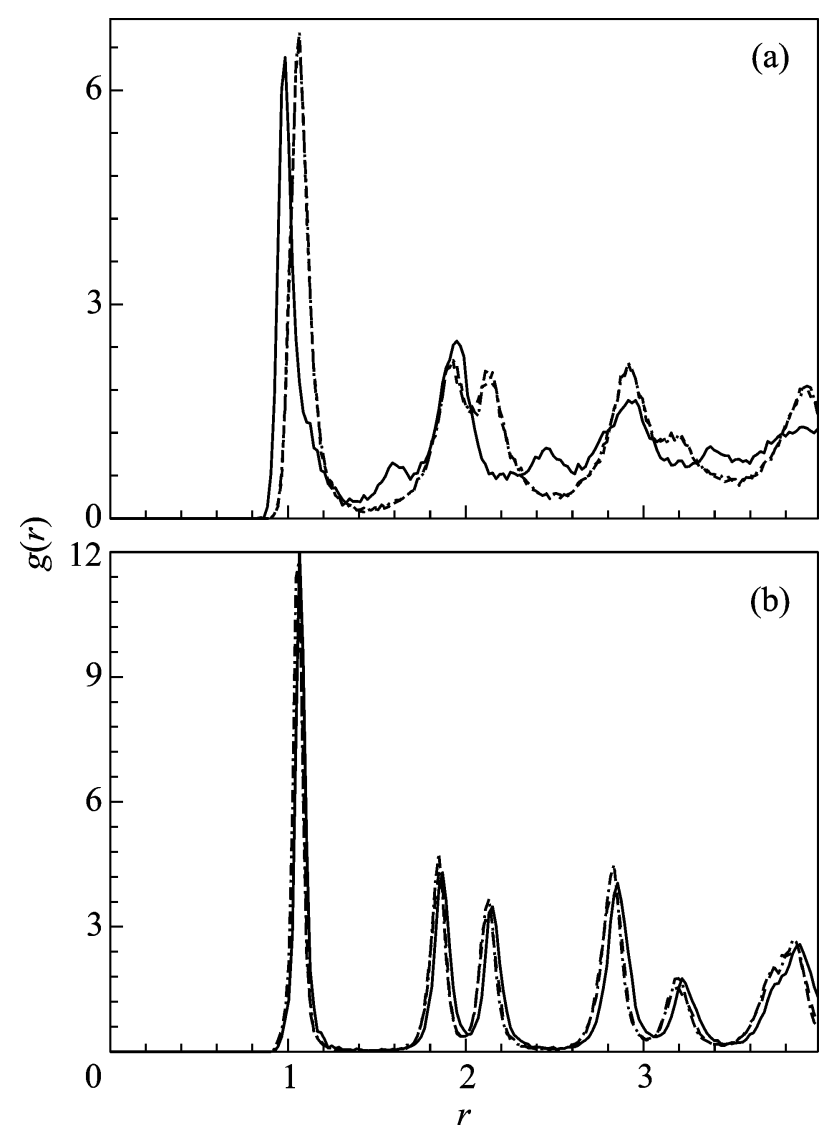

Fig. 5. Pair correlation function $g(r)$ of the three-layer Yukawa system in the (solid line) central, (dashed line) upper, and (dash-dotted line) lower layers for the (a) parabolic and (b) hard wall confinements. The pair correlation functions for the upper and lower layers are almost the same for both confinements.

of a new quasicrystalline phase for the hard wall confinement is very interesting. The unit cell of this phase is shown in the inset in Fig. 3 and is the hop/fcc phase distorted by the rotation of the upper and lower layers with respect to the middle layer.

Figure 4 shows the particle density distribution and the total number of particles in each layer for two confinements. It is seen that, as in the two-dimensional case [24], the density of the microparticles for the hard wall confinement near the boundaries is higher than that in the center. The relation is opposite for the parabolic confinement. Such a density distribution is the main cause of the indicated features of the crystallization.

Figure 5 shows the pair correlation functions $g(r)$ (radial distribution function of the microparticles averaged over all particles in a layer) the three-layer Yukawa system of the microparticles in the (solid line) central, (dashed line) upper, and (dash-dotted line) lower layers for the (a) parabolic and (b) hard elastic wall confinements. The pair correlation functions for the upper and lower layers are almost the same for both confinements. Note that the function $g(r)$ for the central

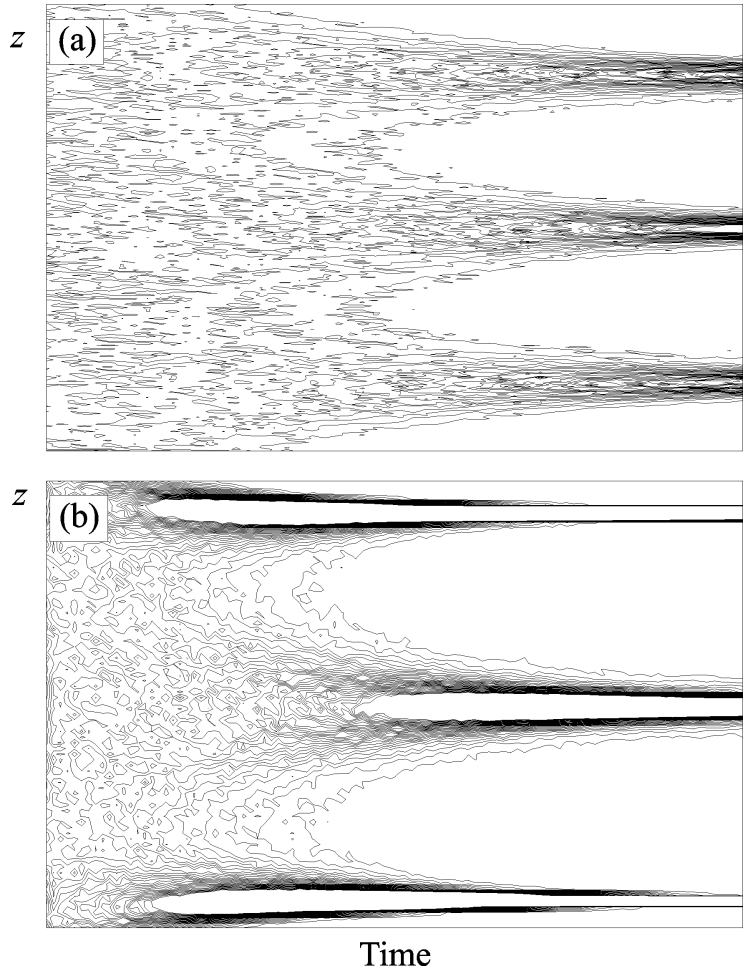

Fig. 6. Space-time $(z-t)$ dependence of the relative microparticle density in the narrow channel for the (a) parabolic and (b) hard wall confinements.

layer is strongly different from this function for the neighboring layers in the case of the parabolic confinement. The shift of the first maximum of $g(r)$ clearly indicates that the particle density in the central layer is higher than that in the neighboring layers for the case of the parabolic confinement. Figure 6 shows the time dependence of the microparticle density for the (a) parabolic and (b) hard wall confinements. In both cases, the formation of three layers from the microparticles randomly distributed at the initial time is seen. For the case of the hard wall confinement, the particles are first crystallized near the boundary $\left(z=0, L_{z}\right)$, whereas all three layers are formed almost simultaneously for the parabolic confinement. Thus, the confinement type noticeably affects the local order and the crystal-lattice type of system of the microparticles whose pair interaction is described by the Yukawa potential in narrow channels. Note that the confinement leads to the appearance of new types of a crystal lattice (face centered, quasicrystalline, and icosahedral phases), which are absent on the phase diagram of the Yukawa system.

In this work, the effect of the confinement on the local order of the crystallized Yukawa system of particles in three-dimensional narrow channels has been numerically investigated using the molecular dynamics method. The parabolic and hard-wall confinements, which are the "soft" and "hard" confinements, have been considered. These types of confinement lead to 
different behaviors of the microparticle density near the boundaries. This behavior, in turn, strongly affects the local order and the type of the crystal lattice in such a system, in particular, a new stable quasicrystalline phase appears for the hard wall confinement. Thus, the principle possibility of controlling the behavior of the complex plasma in narrow channels is confirmed, which can be very important for the investigations and applications of micro- and nanofluids and nanomaterials.

\section{REFERENCES}

1. V. N. Tsytovich, Phys. Usp. 40, 53 (1997).

2. P. K. Shukla and A. A. Mamun, Introduction to Dusty Plasmas Physics (Institute of Physics, Bristol, 2002).

3. V. E. Fortov et al., Phys. Usp. 47, 447 (2004).

4. S. V. Vladimirov and K. Ostrikov, Phys. Rep. 393, 175 (2004).

5. V. E. Fortov et al., Phys. Rep. 421, 1 (2005).

6. M. Horanyi et al., Rev. Geophys. 42, RG4002 (2004).

7. B. A. Klumov, S. V. Vladimirov, and G. E. Morfill, JETP Lett. 85, 478 (2007).

8. B. A. Klumov, S. I. Popel, and G. E. Morfill, JETP 100, 152 (2005).

9. B. A. Klumov, S. V. Vladimirov, and G. E. Morfill, JETP Lett. 82, 632 (2005).
10. G. E. Morfill et al., Phys. Rev. Lett. 92, 175004 (2004).

11. M. Rubin-Zuzic at al., Nature Phys. 2, 181 (2006).

12. B. A. Klumov, M. Rubin-Zuzic, and G. E. Morfill, JETP Lett. 84, 542 (2006).

13. Lee-Wen Teng, Pei-Shan Tu, and Lin I, Phys. Rev. Lett. 90, 245004 (2003).

14. R. D. Deegan et al., Nature 389 (6653), 827 (1997).

15. H. K. Christenson, J. Phys.: Condens. Matter. 13, R95R133 (2001).

16. C. Alba-Simionesco et al., J. Phys.: Condens. Matter. 18, R15-R68 (2006).

17. L. A. Pozhar, Phys. Rev. E 61, 1432 (2000).

18. D. A. Doyle et al., Science 280, 69, 5360 (1998).

19. M. G. Clerk et al., Nature Physics (in press), doi:10.1038/nphys884 (2008).

20. J. H. Chu and Lin I, Phys. Rev. Lett. 72, 4009 (1994).

21. H. Thomas et al., Phys. Rev. Lett. 73, 652 (1994).

22. H. Ikezi, Phys. Fluids 29, 1764 (1986).

23. U. Konopka, G. Morfill, and L. Ratke, Phys. Rev. Lett. 84, 891 (2000).

24. B. A. Klumov and G. E. Morfill, JETP Lett. 85, 498 (2007).

25. S. Hamaguchi, R. T. Farouki, and D. H. E. Dubin, Phys. Rew. E 56, 4671 (1997).

Translated by R. Tyapaev 